\title{
TRUNCATED OPERADS AND SIMPLICIAL SPACES
}

\author{
MICHAEL S. WEISS
}

\begin{abstract}
It was shown in [1] \$7] that a well-known construction which to a (monochromatic, symmetric) topological operad associates a topological category and a functor from it to the category of finite sets is homotopically fully faithful, under mild conditions on the operads. The main result here is a generalization of that statement to $k$-truncated topological operads. A $k$-truncated operad is a weaker version of operad where all operations have arity $\leq k$.
\end{abstract}

\section{INTRODUCTION}

There is a well known construction which to a (monochromatic, symmetric) topological operad $P$ associates a so-called PROP, a small topological category with a symmetric monoidal product. The set of objects of the PROP is identified with the set of natural numbers and the monoidal product corresponds to addition. See [9] for more details and explanations. Forgetting the monoidal product in the PROP and passing to the comma category of objects over 1 produces a topological category (category object in the category of spaces) called $\mathcal{C}_{P}$ in [1, §7]. This comes with a distinguished functor to Fin, essentially the category of all finite sets. It is well known that $P$ can be reconstructed from the associated PROP with the monoidal structure. By contrast, the construction $P \mapsto \mathcal{C}_{P}$ has a forgetful character, even if we include the reference functor $\mathcal{C}_{P} \rightarrow$ Fin, and there are elementary examples to illustrate that it really does forget essential features [1, rem.7.3]. Write $N$ for the nerve construction (from small topological categories to simplicial spaces). The main result of [1, $\S 7]$ is that for topological operads $P$ and $Q$, the map

$$
\mathbb{R} \operatorname{map}(P, Q) \longrightarrow \mathbb{R m a p}_{N \text { Fin }}\left(N \mathcal{C}_{P}, N \mathcal{C}_{Q}\right)
$$

of derived mapping spaces induced by the construction $P \mapsto N \mathcal{C}_{P}$ is nevertheless a weak equivalence under a reasonable condition on $P$ and $Q$. The condition is that the spaces of 0 -ary and 1-ary operations for both $P$ and $Q$ are weakly contractible. (Beware that topological operads with an empty space of 0-ary operations do not qualify, although they are popular.) More details and an example are given in section 2

This paper here relies on [1, §7], but in doing so develops a slightly different proof of the same result. The advantage of the new proof is that it carries over without essential change to the setting of $k$-truncated topological operads. (A $k$-truncated operad is a weaker version of operad which has operations of arity $\leq k$ only.)

2000 Mathematics Subject Classification. 57R40, 55U40, 55P48.

The work was supported by the Bundesministerium für Bildung und Forschung through the A.v.Humboldt foundation (Humboldt professorship 2012-2017). 


\section{OPERADS, DENDROIDAL SPACES AND SIMPLICIAL SPACES}

This section is a review of the main definitions and results of [1, $\S 7$ ].

Let $P$ be an operad in the symmetric monoidal category of spaces. For convenience, the category of spaces is understood to be the category of simplicial sets. The following paragraph in quotation marks is quoted verbatim from [1, §7].

"We think of this in the following terms: $P$ is a functor from the category of finite sets and bijections to spaces, and for every map $f: T \rightarrow S$ of finite sets there is an operation

$$
\lambda_{f}: P(S) \times \prod_{i \in S} P\left(T_{i}\right) \longrightarrow P(T)
$$

where $T_{i}=f^{-1}(i) \subset T$. Also $P(S)$ contains a distinguished unit element when $S$ is a singleton. Sensible naturality, associativity and unital properties are satisfied. Note in particular that any permutation $f: S \rightarrow S$ induces a map $P(S) \rightarrow P(S)$ in two ways: firstly because $P$ is a functor from the category of finite sets and bijections to spaces, and secondly by

$$
P(S) \ni x \mapsto \lambda_{f}(x, 1,1, \ldots, 1) \in P(S) .
$$

for $x \in P(S)$. These two maps agree as per definition.

What we have described is also called a plain operad in the category of spaces ..."

Note that plain is synonymous with monochromatic. It means that the operad has only one object. (We may think of $P(S)$ as the space of $S$-ary operations from that object to itself.) The fact that we allow arbitrary finite sets without a total ordering means that we are dealing with a symmetric operad. In the following we just write operad to mean monochromatic symmetric operad.

There is a construction $P \mapsto \mathcal{C}_{P}$ taking an operad $P$ as above to a topological category $\mathcal{C}_{P}$ (category object in the category of spaces). The category $\mathcal{C}_{P}$ comes with a forgetful functor to the category of finite sets; more precisely we use a skeleton Fin of the category of finite sets. The objects of Fin are the sets $\underline{k}=\{1,2, \ldots, k\}$ where $k \geq 0$. (Note in particular that $\underline{0}$ is the empty set.) The morphisms in Fin from $\underline{k}$ to $\underline{\ell}$ are the maps from $\underline{k}$ to $\underline{\ell}$.

The space of objects of $\mathcal{C}_{P}$ is

$$
\coprod_{k \geq 0} P(\underline{k}) .
$$

The space of morphisms in $\mathcal{C}_{P}$ lifting a morphism $f: \underline{k} \rightarrow \underline{\ell}$ in Fin is

$$
P(\underline{\ell}) \times \prod_{i \in \underline{\ell}} P\left(\underline{k}_{i}\right)
$$

where $k_{i}$ is the cardinality of $f^{-1}(i)$. Source and target of an element in that space are determined by applying to it $\lambda_{f}$ and the projection to $P(\ell)$, respectively. Composition and identity morphisms are obvious. See [1, Rem.7.3] for the relationship between the construction $P \mapsto \mathcal{C}_{P}$ and a better known construction [9] which turns an operad into a PROP.

The main result of [1, §7] states that the construction $P \mapsto \mathcal{C}_{P}$ is homotopically fully faithful as long as it is only used with operads $P$ for which the spaces $P(\underline{0})$ and $P(\underline{1})$ are weakly contractible. Making this precise requires a few decisions. Clearly $P \mapsto \mathcal{C}_{P}$ is a functor. But we need to reason about derived spaces of morphisms between two topological operads, or between two small topological 
categories. Therefore it is convenient to work with a preferred model category of topological operads, and with a preferred model category of small topological categories.

Rezk has introduced a model category setting for the category of small topological categories. The underlying category is simply the category of simplicial spaces (and here space still means simplicial set for us). A simplicial space $X$ is said to be a Segal space if it satisfies the following condition $(\sigma)$. Let $u_{i}:\{0,1\} \rightarrow\{0,1,2, \ldots, n\}$ be the order-preserving map defined by $u_{i}(0)=i-1$ and $u_{i}(1)=i$.

$(\sigma)$ For each $n \geq 2$ the map $\left(u_{1}^{*}, u_{2}^{*}, \ldots, u_{n}^{*}\right)$ from $X_{n}$ to the homotopy limit of the diagram

$$
X_{1} \stackrel{d_{0}}{\longrightarrow} X_{0} \stackrel{d_{1}}{\longleftarrow} X_{1} \stackrel{d_{0}}{\longrightarrow} \cdots \quad \cdots \stackrel{d_{0}}{\longrightarrow} X_{0} \stackrel{d_{1}}{\longleftarrow} X_{1}
$$

is a weak homotopy equivalence.

Examples: the nerve $N \mathcal{C}$ of a small category $\mathcal{C}$, which is a simplicial set and as such a simplicial space, is a Segal space. (Note in passing that the set of $r$-simplices of $N \mathcal{C}$ is the set of contravariant functors from $I_{r}$ to $\mathcal{C}$, where $I_{r}$ is $\{0,1, \ldots, r\}$ viewed as a poset with the usual ordering.) The nerve of a small topological category $\mathcal{C}$ is a Segal space provided that at least one of the maps source, target from the space of morphisms of $\mathcal{C}$ to the space of objects of $\mathcal{C}$ is a (Kan) fibration.

For a Segal space $X$ it is sensible to view $X_{0}$ as the space of objects of something slightly more general than a topological category, and to view $X_{1}$ as the space of morphisms, and $d_{0}, d_{1}: X_{1} \rightarrow X_{0}$ as the operators source and target, respectively. In the same spirit, let

$$
\operatorname{mor}_{X}^{h}(a, b):=\operatorname{hofiber}_{(a, b)}\left[\left(d_{0}, d_{1}\right): X_{1} \rightarrow X_{0} \times X_{0}\right]
$$

for $a, b \in X_{0}$. There is a composition map $\operatorname{mor}_{X}^{h}(b, c) \times \operatorname{mor}_{X}^{h}(a, b) \rightarrow \operatorname{mor}_{X}^{h}(a, c)$, well defined at least up to homotopy.

A simplicial map $f: X \rightarrow Y$ between simplicial spaces which satisfy $(\sigma)$ is a DwyerKan equivalence if

- for every $a, b \in X_{0}$ the map

$$
\operatorname{mor}_{X}^{h}(a, b) \longrightarrow \operatorname{mor}_{Y}^{h}(f(a), f(b))
$$

induced by $f$ is a weak equivalence;

- essential surjectivity: for every $c \in Y_{0}$ there exist $b \in X_{0}$ and an element of $\operatorname{mor}_{Y}^{h}(f(b), c)$ which is weakly invertible.

A simplicial space $X$ is a complete Segal space if in addition to $(\sigma)$ it satisfies another property $(\kappa)$, completeness, for which the reader can consult [1, §2]. The point of this additional condition is the following. Firstly, every Segal space admits a DwyerKan equivalence to a complete Segal space. Secondly, a Dwyer-Kan equivalence between two Segal spaces which are both complete is a degreewise weak equivalence.

Rezk's model category structure on the category $s \mathcal{S}$ of simplicial spaces can be described roughly as follows. Start with the standard model category structure on $\mathcal{S}$, the category of spaces (=simplicial sets). Use it to define a model category structure on $s \mathcal{S}$ where either the weak equivalences and the cofibrations are defined levelwise, or the weak equivalences and the fibrations are defined levelwise (yes, there are two options). Write $s \mathcal{S}_{1}$ for $s \mathcal{S}$ with this model category structure. There is a unique model category structure on $s \mathcal{S}$ which has the same cofibrations as $s \mathcal{S}_{1}$ and which for the fibrant objects has the complete Segal spaces which are also 
fibrant as objects of $s \mathcal{S}_{1}$. See [1, §B.2]. This is the Rezk model category structure. Write $s \mathcal{S}_{2}$ for $s \mathcal{S}$ with that model category structure. A map $f: X \rightarrow Y$ of simplicial spaces is a weak equivalence in $s \mathcal{S}_{2}$ if and only if, for every complete Segal space $Z$ which is fibrant in $s \mathcal{S}_{1}$, the induced map

$$
\mathbb{R m a p}_{s \mathcal{S}_{1}}(Y, Z) \rightarrow \mathbb{R m a p}_{s \mathcal{S}_{1}}(X, Z)
$$

(of derived mapping spaces formed in $s \mathcal{S}_{1}$ ) is a weak equivalence. This characterization of the weak equivalences means that $s \mathcal{S}_{2}$ can also be constructed from $s \mathcal{S}_{1}$ by a left localization.

We will also need a model category structure on the over category $s \mathcal{S} / Z$ where $Z$ is an object of $s \mathcal{S}$, for us typically a Segal space but not a complete one. There is a standard way by which a model category structure on some category $\mathcal{C}$ determines a model category structure on each of the over categories $\mathcal{C} / c$, for objects $c$ of $\mathcal{C}$. See [6. Expl.1.7]. We apply this with $s \mathcal{S}_{2}$ and we denote the result by $s \mathcal{S}_{2} / Z$. By definition the fibrant objects in $s \mathcal{S}_{2} / Z$ are simply the fibrations with target $Z$ in $s \mathcal{S}_{2}$. But they also have a characterization as the morphisms $Y \rightarrow Z$ in $s \mathcal{S}$ which are fibrations in $s \mathcal{S}_{1}$ and make $Y$ into a fiberwise complete Segal space over $Z$; see 11, $\S \mathrm{B}]$ for more details.

Cisinski and Moerdijk 3 . have an analogous framework for topological operads; see also related earlier papers by Cisinski and Moerdijk [2, and by Moerdijk and I.Weiss [7. The starting point for this is a small category Tree (in their notation, $\Omega$ ) whose objects are certain finite trees. In more detail, an object $T$ of Tree is a finite nonempty set $\epsilon(T)$ with a partial order $\leq$ and a distinguished subset $\lambda(T)$ of the set of maximal elements of $\epsilon(T)$ such that the following conditions are satisfied:

- $\epsilon(T)$ has a minimal element (called the root);

- for each element $e$ of $\epsilon(T)$, the set $\{y \in \epsilon(T) \mid y \leq e\}$ with the restricted ordering is linearly ordered.

The elements of $\epsilon(T)$ are also called edges of the tree $T$. The elements of $\lambda(T)$ are called the leaves of $T$. (The elements of $\epsilon(T) \backslash \lambda(T)$ are sometimes called vertices, but I have found this confusing and I prefer to call them non-leaf edges.) An object $T$ of Tree generates a finite (colored!) operad with color set $\epsilon(T)$; for each non-leaf edge $a$ in $T$ there is a generating operation with target $a$ and with multi-source equal to the set of edges which are just above $a$ in the ordering. A morphism $S \rightarrow T$ in Tree is by definition a morphism of the associated finite operads. See [1. $\S 7.2]$ for more details and examples. The category Tree contains a copy of the category $\Delta$ (with objects $[k]=\{0,1, \ldots, k\}$ for $k \geq 0$ and with monotone maps as morphisms). A dendroidal space is a functor from Tree ${ }^{\mathrm{op}}$ to spaces. Therefore a dendroidal space determines a simplicial space by restriction from Tree ${ }^{\mathrm{op}}$ to $\Delta^{\mathrm{op}}$. There is a concept of Segal dendroidal space analogous to the concept of Segal space, and a corresponding model category structure on the category $d \mathcal{S}$ of dendroidal spaces. Again, this can be obtained by starting with a standard model category structure on $d \mathcal{S}$, for which we write $d \mathcal{S}_{1}$, and then applying a localization process. The result is $d \mathcal{S}_{2}$ which has the same underlying category and the same cofibrations as $d \mathcal{S}_{1}$, but the fibrant objects in $d \mathcal{S}_{2}$ are the Segal dendroidal spaces which are also fibrant in $d \mathcal{S}_{1}$.

A topological operad $Q$ has a dendroidal nerve $N_{d} Q$, which is a dendroidal space such that $\left(N_{d} Q\right)_{T}$ is the space of operad maps from the operad associated with the tree $T$ to $Q$. If $Q$ is monochromatic, then $\left(N_{d} Q\right)_{T}$ is a point for $T=\eta$, the tree 
with a single edge. (The notation $N_{d} Q$ for the dendroidal nerve of an operad $Q$ is widely used. It clashes with the notation $N_{d} \mathcal{C}$ for the set of $d$-simplices in the nerve of a category $\mathcal{C}$. Notation like $(N \mathcal{C})_{d}$ can be used in such cases.) The dendroidal nerve $N_{d} Q$ is then a Segal dendroidal space. (It does not make sense to insist on a completeness property as in [8, or to claim such a property, if we want to work with monochromatic operads. I am indebted to the referee for drawing my attention to this point. By analogy, the bar construction alias nerve of a topological monoid $M$ is a simplicial space which qualifies as a Segal space, but it is a complete Segal space only if the subspace of weakly invertible elements in $M$ is weakly contractible. See also [1, Expl.7.7]. Similarly, if the monochromatic operad $Q$ satisfies $Q(1) \simeq *$, then $N_{d} Q$ can call itself a complete Segal dendroidal space, but we are not imposing that condition yet.) A key result of the dendroidal theory is that the dendroidal nerve functor induces a weak equivalence

$$
\mathbb{R} \operatorname{map}(P, Q) \stackrel{\simeq}{\longrightarrow} \mathbb{R} \operatorname{map}\left(N_{d} P, N_{d} Q\right)
$$

when $P$ and $Q$ are (monochromatic) operads. In the left-hand side, the derived mapping space $\mathbb{R} \operatorname{map}(P, Q)$ can be interpreted using a standard model structure with levelwise weak equivalences and fibrations. In the right-hand side, use either $d \mathcal{S}_{1}$ or $d \mathcal{S}_{2}$; it does not matter which because $N_{d} Q$ is a Segal dendroidal space.

For a simplicial set $Y$ let $\operatorname{simp}(Y)$ be the category whose objects are pairs $(m, y)$ with $y \in X_{m}$; a morphism from $(m, y)$ to $(n, z)$ is a morphism $f:[m] \rightarrow[n]$ in $\Delta$ such that $f^{*}(z)=y$. There is a functor

$$
\varphi: \operatorname{simp}(N \mathrm{Fin}) \longrightarrow \text { Tree }
$$

defined as follows. To an object $\left(p, S_{*}\right)$ of $\operatorname{simp}(N F i n)$, where

$$
S_{*}=\left(S_{0} \leftarrow S_{1} \leftarrow S_{2} \leftarrow \cdots \leftarrow S_{p}\right)
$$

associate the tree $T$ where $\epsilon(T)$ is the disjoint union of the $S_{i}$ and an additional element $r$, with $\lambda(T)$ corresponding to $S_{p}$. The partial order on $\epsilon(T)$ is the obvious one: $r$ is the minimal element and $x \in S_{i}$ is $\leq y \in S_{j}$ if $i \leq j$ and the composite map from $S_{j}$ to $S_{i}$ in the string $S_{*}$ takes $y$ to $x$. The functor $\varphi$ establishes up a close relationship between dendroidal spaces and simplicial spaces over $N$ Fin. Indeed, a simplicial space over NFin is the same thing as a contravariant functor from $\operatorname{simp}(N$ Fin $)$ to spaces. Therefore pre-composition with $\varphi$ is a functor $\varphi^{*}$ which takes us from dendroidal spaces to simplicial spaces over NFin. Important example: for a topological operad there is an isomorphism of $\varphi^{*}\left(N_{d} P\right)$ with $N \mathcal{C}_{P}$, both viewed as simplicial spaces over NFin.

The main result of [1, $\S 7]$ is that for topological operads $P$ and $Q$ the functor $\varphi^{*}$ induces a weak equivalence

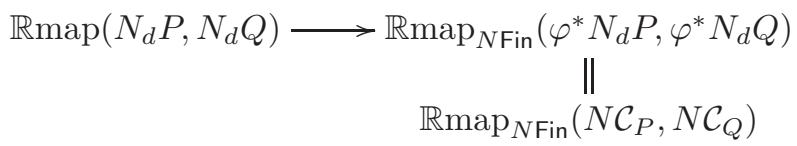

provided $P(\underline{0}), P(\underline{1}), Q(\underline{0})$ and $Q(\underline{1})$ are weakly contractible. In the right-hand side we can use the model category structure $s \mathcal{S}_{1} / N$ Fin or $s \mathcal{S}_{2} / N$ Fin ; it does not matter because $N \mathcal{C}_{Q}$ is a fiberwise complete Segal space over $N$ Fin. - In view of (2.1) 
this implies that the nerve functor determines a weak equivalence

$$
\mathbb{R} \operatorname{map}(P, Q) \stackrel{\simeq}{\longrightarrow} \operatorname{Rmap}_{N \text { Fin }}\left(N \mathcal{C}_{P}, N \mathcal{C}_{Q}\right)
$$

under the same conditions on $P$ and $Q$.

Example 2.1. Suppose that $P$ is the operad of little $m$-disks and $Q$ is the operad of little $n$-disks. Then $N \mathcal{C}_{P}$ and $N \mathcal{C}_{Q}$ are weakly equivalent, over $N$ Fin, to certain simplicial spaces con $\left(\mathbb{R}^{m}\right)$ and $\operatorname{con}\left(\mathbb{R}^{n}\right)$ defined as nerves of certain (topological) categories of configurations in $\mathbb{R}^{m}$ and $\mathbb{R}^{n}$, respectively. See [1, §3] and [1, expl.7.2]. With Andrade's particle models for the configuration categories, $M \mapsto \operatorname{con}(M)$ is a continuous functor on the category of topological manifolds and injective continuous maps. It follows that there are compatible actions of the homeomorphism group of $\mathbb{R}^{m}$ (from the right) and the homeomorphism group of $\mathbb{R}^{n}$ (from the left) on

$$
\mathbb{R m a p}_{N \text { Fin }}\left(\operatorname{con}\left(\mathbb{R}^{m}\right), \operatorname{con}\left(\mathbb{R}^{n}\right)\right) \simeq \mathbb{R m a p}_{N \text { Fin }}\left(N \mathcal{C}_{P}, N \mathcal{C}_{Q}\right) .
$$

Similarly, there is an interesting map from the space of injective continuous maps $\mathbb{R}^{m} \rightarrow \mathbb{R}^{n}$ to the space $\mathbb{R m a p}_{N \text { Fin }}\left(\operatorname{con}\left(\mathbb{R}^{m}\right), \operatorname{con}\left(\mathbb{R}^{n}\right)\right)$. All these good features are not easy to see in the operadic description $\mathbb{R} \operatorname{map}(P, Q)$.

\section{TRuncated operads And TRUnCATED DENDRoidal SPACES}

For an integer $k \geq 1$, a $k$-truncated operad in the symmetric monoidal category of spaces is defined like an operad in spaces except for the following changes: all operations have arity $\leq k$ and composition of operations is only defined where it does not contradict this restriction. More specifically, we can describe a $k$-truncated (monochromatic, symmetric) operad in spaces in the following terms. It is a functor $P$ from the category of finite sets of cardinality $\leq k$ and bijections to spaces, and for every map $f: T \rightarrow S$ of finite sets of cardinality $\leq k$ there is an operation

$$
\lambda_{f}: P(S) \times \prod_{i \in S} P\left(T_{i}\right) \longrightarrow P(T)
$$

where $T_{i}=f^{-1}(i) \subset T$. Also $P(S)$ contains a distinguished unit element when $S$ is a singleton. Sensible naturality, associativity and unital properties are satisfied. In particular any permutation $f: S \rightarrow S$, where $|S| \leq k$, induces a map $P(S) \rightarrow P(S)$ in two ways: firstly because $P$ is a functor, and secondly by

$$
P(S) \ni x \mapsto \lambda_{f}(x, 1,1, \ldots, 1) \in P(S) .
$$

for $x \in P(S)$. These two maps agree as per definition.

For an integer $k \geq 1$ let Tree $_{k} \subset$ Tree be the full subcategory whose objects are the trees $T$ such that for every $t \in \epsilon(T) \backslash \lambda(T)$ the set $\{s \in \epsilon(T) \mid s>t\}$ has at most $k$ minimal elements. (These minimal elements are often called the incoming edges ... of a fictional vertex associated with the non-leaf edge $t$.) Similarly let Fin $\leq k$ be the full subcategory spanned by the objects $\underline{m}$ where $m \leq k$. The functor $\varphi$ above restricts to a functor

$$
\varphi_{k}: \operatorname{simp}\left(N \mathrm{Fin}_{\leq k}\right) \longrightarrow \text { Tree }_{k} .
$$

An object $T$ of Tree $k$ generates a finite $k$-truncated (colored) operad with color set $\epsilon(T)$; for each non-leaf edge $a$ in $T$ there is a generating operation with target $a$ and with multi-source equal to the set of edges which are just above $a$ in the ordering. A $k$-truncated operad $P$ has a nerve $N_{d} P$ which we regard as a contravariant functor 
from Tree $_{k}$ to spaces. It is defined in such a way that $\left(N_{d} P\right)_{T}$ is the space of $k$ truncated operad morphisms from the $k$-truncated operad associated with $T$ to $P$. The $k$-truncated operad $P$ also determines a topological category $\mathcal{C}_{P}$ (category in spaces) with a forgetful functor to $\mathrm{Fin}_{\leq k}$. The space of objects of $\mathcal{C}_{P}$ is

$$
\coprod_{m=0}^{k} P(\underline{m}) \text {. }
$$

For a morphism $f: \underline{\ell} \rightarrow \underline{m}$ in Fin $\leq k$ the space of morphisms in $\mathcal{C}_{P}$ lifting $f$ is

$$
P(\underline{\ell}) \times \prod_{j \in \underline{m}} P\left(\underline{\ell}_{j}\right)
$$

where $\ell_{j}$ is the cardinality of $f^{-1}(j)$.

Keeping in mind that a simplicial space over $N$ Fin ${ }_{\leq k}$ is the same thing as a contravariant functor from $\operatorname{simp}(N$ Fin $\leq k)$, we can say that composition with $\varphi_{k}$ is a functor $\varphi_{k}^{*}$ from $k$-truncated dendroidal spaces to $\operatorname{simplicial~spaces~over~} N$ Fin $_{\leq k}$. In that sense there is an isomorphism

$$
\varphi_{k}^{*}\left(N_{d} P\right) \cong N \mathcal{C}_{P}
$$

of simplicial spaces over $N \mathrm{Fin}_{\leq k}$, assuming that $P$ is a $k$-truncated operad in spaces.

Theorem 3.1. Let $P$ and $Q$ be $k$-truncated operads (in spaces, $k \geq 1$ ) for which the spaces $P(\underline{0}), P(\underline{1}), Q(\underline{0})$ and $Q(\underline{1})$ are weakly contractible. Then composition with the functor $\varphi_{k}^{*}$ is a weak equivalence

$$
\begin{array}{r}
\mathbb{R m a p}\left(N_{d} P, N_{d} Q\right) \stackrel{\simeq}{\longrightarrow} \operatorname{Rmap}_{N \mathrm{Fin}_{\leq k}}\left(\varphi_{k}^{*} N_{d} P, \varphi^{*} N_{d} Q\right) \\
{\mathbb{R} \operatorname{map}_{N \mathrm{Fin}_{\leq k}}\left(N \mathcal{C}_{P}, N \mathcal{C}_{Q}\right) .}^{\|}
\end{array}
$$

This will be proved in the following sections. For clarification, we make sense of $\mathbb{R} \operatorname{map}\left(N_{d} P, N_{d} Q\right)$ using a model category structure with levelwise weak equivalences on the category of contravariant functors from Tree $_{k}$ to spaces. We make sense of $\mathbb{R m a p}_{N \text { Fin } \leq k}\left(N \mathcal{C}_{P}, N \mathcal{C}_{Q}\right)$ using a model category structure on the category of simplicial spaces over $N$ Fin $\leq k$ with levelwise weak equivalences. (See [5], where it is shown that the derived mapping spaces in a model category depend mainly on the subcategory of weak equivalences, not much on the subcategories of cofibrations and fibrations, respectively.) The proof of theorem 3.1 given here works equally well in the untruncated setting, $k=\infty$. It can be seen as another way to show that (2.2) is a weak equivalence which happens to generalize easily to the truncated situation.

\section{LEAVES ARE UNNECESSARY}

In [1, §7] the weak equivalence (2.2) is established in essentially two steps. In the first step, which is the easier one, the category Tree gets replaced by a much more accessible subcategory Tree ${ }^{\text {rc }}$. It is the subcategory of Tree obtained by allowing as objects only the trees $T$ with empty leaf set $\lambda(T)$ and as morphisms only the morphisms in Tree between trees with no leaves which take root to root.

An object $T$ of Tree ${ }^{r c}$ can therefore be described as a finite set $T$ with an order relation (written $\leq$ ) such that

- $T$ has a (unique) minimal element, called the root; 
- for every $s \in T$ the set $\{t \in T \mid t \leq s\}$ is linearly ordered (with the ordering induced from $T$ ).

(We need not distinguish anymore between $T$ and the edge set of $T$.) A morphism $S \rightarrow T$ in Tree ${ }^{r c}$ is a map $f$ from $S$ to $T$ which respects the order relations, takes root to root and has the additional property that it preserves independence. That is, if for $u, v \in S$ we have neither $u \leq v$ nor $v \leq u$, then for $f(u), f(v) \in T$ we have neither $f(u) \leq f(v)$ nor $f(v) \leq f(u)$.

There is a functor $\psi: \operatorname{simp}(N$ Fin $) \rightarrow$ Tree $^{\text {rc }}$ very similar to $\varphi: \operatorname{simp}(N$ Fin $) \rightarrow$ Tree. For an object $\left(p, S_{*}\right)$ of $\operatorname{simp}(N$ Fin $)$, where

$$
S_{*}=\left(S_{0} \leftarrow S_{1} \leftarrow S_{2} \leftarrow \cdots \leftarrow S_{p}\right),
$$

let $\psi\left(p, S_{*}\right)$ be tree $T$ without leaves which, as a set, is the disjoint union of the $S_{i}$ and an additional element $r$. The partial order on $T$ is the obvious one: $r$ is the minimal element and $x \in S_{i}$ is $\leq y \in S_{j}$ if $i \leq j$ and the composite map from $S_{j}$ to $S_{i}$ in the string $S_{*}$ takes $y$ to $x$.

The formal relationship between $\varphi$ and $\psi$ is a little more complicated than one might expect. The inclusion $\iota:$ Tree $^{r c} \rightarrow$ Tree has a left adjoint $\kappa:$ Tree $\rightarrow$ Tree ${ }^{r c}$. Equations such as $\varphi=\iota \psi$ or $\psi=\kappa \varphi$ come to mind, but both are false. Instead we have $\varphi \beta=\iota \psi$, where $\beta: \operatorname{simp}(N$ Fin $) \rightarrow \operatorname{simp}(N$ Fin $)$ is the endofunctor which takes a string

to the string

$$
\left(S_{0} \leftarrow S_{1} \leftarrow S_{2} \leftarrow \cdots \leftarrow S_{p}\right)
$$

$$
\left(S_{0} \leftarrow S_{1} \leftarrow S_{2} \leftarrow \cdots \leftarrow S_{p} \leftarrow \emptyset\right) .
$$

Now let $N_{d}^{r c} P$ and $N_{d}^{r c} Q$ be the restrictions of $N_{d} P$ and $N_{d} Q$, respectively, to Tree $^{r c}$. On the basis of the observations just above, showing that (2.2) is a weak equivalence reduces easily to showing that the map

$$
\mathbb{R} \operatorname{map}\left(N_{d}^{r c} P, N_{d}^{r c} Q\right) \longrightarrow \mathbb{R} \operatorname{map}\left(\psi^{*} N_{d}^{r c} P, \psi^{*} N_{d}^{r c} Q\right)
$$

obtained by composition with $\psi^{*}$ is a weak equivalence. We are still assuming that $P(\underline{0}), P(\underline{1})$ and $Q(\underline{0}), Q(\underline{1})$ are weakly contractible. (There is a commutative diagram

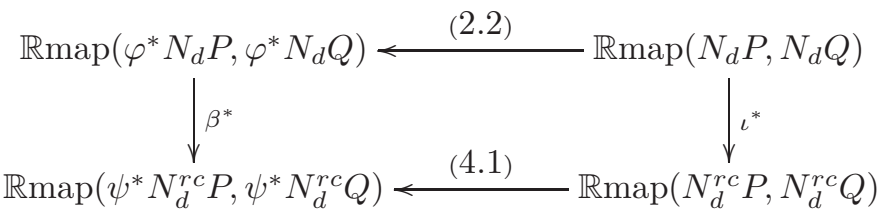

where the vertical arrows are weak equivalences; use $\varphi \beta=\iota \psi$ and $N_{d}^{r c}=\iota^{*} N_{d}$.)

The message of this short section is that the same argument applies in the truncated situation. There is a functor

$$
\psi_{k}: \operatorname{simp}\left(N \mathrm{Fin}_{\leq k}\right) \longrightarrow \text { Tree }_{k} \cap \text { Tree }^{\text {rc }}
$$

obtained by restriction of $\psi$. Suppose that $P$ and $Q$ are $k$-truncated operads for which $P(\underline{0}), P(\underline{1})$ and $Q(\underline{0}), Q(\underline{1})$ are weakly contractible. Let $N_{d}^{r c} P$ and $N_{d}^{r c} Q$ be the contravariant functors from Tree $_{k} \cap$ Tree $^{r c}$ to spaces obtained by restricting $N_{d} P$ and $N_{d} Q$, respectively. Then for the proof of theorem 3.1 it suffices to show that the map

$$
\mathbb{R} \operatorname{map}\left(N_{d}^{r c} P, N_{d}^{r c} Q\right) \longrightarrow \mathbb{R} \operatorname{map}\left(\psi_{k}^{*} N_{d}^{r c} P, \psi_{k}^{*} N_{d}^{r c} Q\right)
$$


obtained by composing with $\psi_{k}^{*}$ is a weak equivalence.

\section{BRIDGING THE GAP}

Let $\mathbb{R} \psi_{*}$ be the homotopy right Kan extension along $\psi$. This is applicable to contravariant functors from $\operatorname{simp}(N$ Fin $)$ to spaces and yields contravariant functors from Tree ${ }^{r c}$ to spaces. It serves as a homotopy right adjoint to the functor $\psi^{*}$ given by pre-composition with $\psi$. It is shown in [1, §7] that, under conditions on $Q$ as in (4.1), the homotopy unit

$$
N_{d}^{r c} Q \longrightarrow \mathbb{R} \psi_{*} \psi^{*}\left(N_{d}^{r c} Q\right)
$$

is a weak equivalence. This implies in a formal manner that the map (4.1) is a weak equivalence. See [1, lem.A.1].

This type of argument is also available in the truncated setting, but showing that the truncated analogue of (5.1) is a weak equivalence is harder than showing that (5.1) is a weak equivalence. Therefore we proceed in two steps by writing the functor $\psi$ and its truncated variant $\psi_{k}$ as a composition of two functors. In doing so we deviate from the line of reasoning developed in 1, §7]. It amounts to additional work, but there is the surprising reward that we can avoid the use of a difficult lemma [1, lem.7.14].

Definition 5.1. There is a category Levtree which is halfway between $\operatorname{simp}(N$ Fin) and Tree ${ }^{r c}$. An object of Levtree is an object of simp(NFin). A morphism in Levtree from $S_{*}=\left(S_{0} \leftarrow S_{1} \leftarrow \cdots \leftarrow S_{k}\right)$ to $R_{*}=\left(R_{0} \leftarrow R_{1} \leftarrow \cdots \leftarrow R_{\ell}\right)$ consists of a monotone map $u:[k] \rightarrow[\ell]$ and monotone injections $v_{j}: S_{j} \rightarrow R_{u(j)}$, one for every $j \in[k]$, such that the diagrams

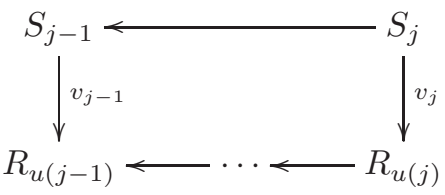

commute for $j \in\{1, \ldots, k\}$. If the $v_{j}$ are bijective, then they are necessarily identity maps and the collection $\left(u,\left(v_{j}\right)\right)$ is a morphism in $\operatorname{simp}\left(N\right.$ Fin) from $S_{*}$ to $R_{*}$. Therefore $\operatorname{simp}(N$ Fin $) \subset$ Levtree.

Let us note that in the category $\operatorname{simp}(N$ Fin) or, for that matter, in any category of the form $\operatorname{simp}(Y)$ where $Y$ is a simplicial set, no object admits nontrivial automorphisms. The same can be said of Levtree: no object admits nontrivial automorphisms.

Write $\alpha: \operatorname{simp}(N$ Fin $) \rightarrow$ Levtree for the inclusion. The functor $\psi$ has an obvious extension to a functor $\xi$ : Levtree $\rightarrow$ Tree $^{\text {rc }}$, so that there is a commutative triangle

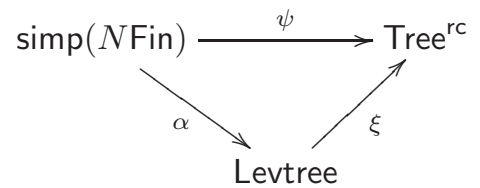

Lemma 5.2. Let $Z=\xi^{*}\left(N_{d}^{r c} Q\right)$. The homotopy unit $Z \rightarrow \mathbb{R} \alpha_{*} \alpha^{*} Z$ is a weak equivalence. 
Proof. Let $R_{*}=\left(R_{0} \leftarrow R_{1} \leftarrow \cdots \leftarrow R_{\ell}\right)$ be an object of Levtree. For $\left(\mathbb{R} \alpha_{*} \alpha^{*} Z\right)\left(R_{*}\right)$ we have the standard formula

$$
\operatorname{holim}_{S_{*} \rightarrow R_{*}} Z\left(S_{*}\right)
$$

where the homotopy limit is taken over a certain comma category or over category $\mathscr{U}$ which depends on $R_{*}$. The objects of $\mathscr{U}$ are pairs consisting of an object $S_{*}$ of $\operatorname{simp}\left(N\right.$ Fin) and a morphism $f: S_{*} \rightarrow R_{*}$ in Levtree. A morphism in $\mathscr{U}$ from $\left(S_{*}, f\right)$ to $\left(S_{*}^{\prime}, g\right)$ is a morphism $S_{*} \rightarrow S_{*}^{\prime}$ in $\operatorname{simp}(N$ Fin $)$ which is over $R_{*}$ when viewed as a morphism in Levtree.

We introduce full subcategories

$$
\mathscr{U}_{-1} \supset \mathscr{U}_{0} \supset \mathscr{U}_{1} \supset \mathscr{U}_{2} \supset \cdots \supset \mathscr{U}_{\ell-1} \supset \mathscr{U}_{\ell}
$$

of $\mathscr{U}$, where $\mathscr{U}_{-1}=\mathscr{U}$ and $\mathscr{U}_{\ell}$ is the comma category determined by the identity functor $\operatorname{simp}(N$ Fin $) \rightarrow \operatorname{simp}(N$ Fin $)$ and the object $R_{*}$. (The integer $\ell$ is determined by $R_{*}$.) The details are as follows. An object of $\mathscr{U}$ given by $S_{*} \rightarrow R_{*}$, or more precisely, by a commutative diagram

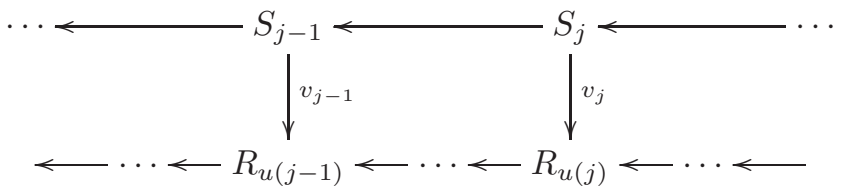

belongs to $\mathscr{U}_{r}$ if and only if $v_{j}$ is bijective (hence an identity map) for all $j$ such that $u(j) \leq r$. In particular that object $S_{*} \rightarrow R_{*}$ belongs to $\mathscr{U}_{\ell}$ if and only if $S_{*} \rightarrow R_{*}$ is a morphism in $\operatorname{simp}\left(N\right.$ Fin). We use the abbreviation $\bar{Z}\left(S_{*} \rightarrow R_{*}\right):=Z\left(S_{*}\right)$ for an object $S_{*} \rightarrow R_{*}$ in $\mathscr{U}$. Then $\bar{Z}$ is a contravariant functor from $\mathscr{U}$ to spaces and

$$
\left(\mathbb{R} \alpha_{*} \alpha^{*} Z\right)\left(R_{*}\right)=\operatorname{holim} \bar{Z}=\operatorname{holim} \bar{Z} \mid \mathscr{U}_{-1} \text {. }
$$

There is a string of forgetful projections

$$
\operatorname{holim} \bar{Z}\left|\mathscr{U}_{-1} \longrightarrow \operatorname{holim} \bar{Z}\right| \mathscr{U}_{0} \longrightarrow \cdots \longrightarrow \operatorname{holim} \bar{Z} \mid \mathscr{U}_{\ell} .
$$

We have our unit map from $Z\left(R_{*}\right)$ to $\left(\mathbb{R} \alpha_{*} \alpha^{*} Z\right)\left(R_{*}\right)=$ holim $\bar{Z} \mid \mathscr{U}_{-1}$ such that the composition

$$
Z\left(R_{*}\right) \rightarrow \operatorname{holim} \bar{Z}\left|\mathscr{U}_{-1} \longrightarrow \operatorname{holim} \bar{Z}\right| \mathscr{U}_{0} \longrightarrow \cdots \longrightarrow \operatorname{holim} \bar{Z} \mid \mathscr{U}_{\ell}
$$

is a weak equivalence for rather trivial reasons. Therefore it suffices to show that each of the projection maps holim $\bar{Z} \mid \mathscr{U}_{r} \rightarrow$ holim $\bar{Z} \mid \mathscr{U}_{r+1}$ admits a homotopy left inverse, making holim $\bar{Z} \mid \mathscr{U}_{r}$ a homotopy retract of holim $\bar{Z} \mid \mathscr{U}_{r+1}$. To achieve that we shall construct two functors

$$
V: \mathscr{U}_{r} \rightarrow \mathscr{U}_{r}, \quad W: \mathscr{U}_{r} \rightarrow \mathscr{U}_{r+1}
$$

and natural transformations id $\Rightarrow V \Leftarrow W$, where the functor $V$ takes $\mathscr{U}_{r+1}$ to itself. The crucial property is that, for every object $S_{*} \rightarrow R_{*}$ in $\mathscr{U}_{r}$, the natural morphism $W\left(S_{*} \rightarrow R_{*}\right) \rightarrow V\left(S_{*} \rightarrow R_{*}\right)$ is taken to a weak equivalence by the functor $\bar{Z}$. Then we shall have the following maps:

$$
\begin{aligned}
& \operatorname{holim} \bar{Z} \mid \mathscr{U}_{r+1} \longrightarrow \operatorname{holim}\left(\bar{Z} \mid \mathscr{U}_{r+1}\right) \circ W \\
& \simeq \uparrow \uparrow \\
& \operatorname{holim}\left(\bar{Z} \mid \mathscr{U}_{r}\right) \circ V \longrightarrow \operatorname{holim} \bar{Z} \mid \mathscr{U}_{r}
\end{aligned}
$$


(the first by prolongation, the other two using the natural transformations) which give us the required homotopy class of maps from holim $\bar{Z} \mid \mathscr{U}_{r+1}$ to holim $\bar{Z} \mid \mathscr{U}_{r}$. For the description of $V$, imagine an object $S_{*} \rightarrow R_{*}$ of $\mathscr{U}_{r}$ given by a diagram like (5.3), where $j$ runs through $\{0,1, \ldots, k\}$. Determine the unique $t \in$ $\{0,1, \ldots, k, k+1\}$ such that $u(j) \leq r$ whenever $j<t$ and $u(j)>r$ whenever $j \geq t$. Let $V\left(S_{*} \rightarrow R_{*}\right)=\left(S_{*}^{\prime} \rightarrow R_{*}\right)$ where

$$
S_{*}^{\prime}=\left(S_{0} \leftarrow S_{1} \leftarrow \cdots \leftarrow S_{t-1} \leftarrow R_{r+1} \leftarrow S_{t} \leftarrow S_{t+1} \leftarrow \cdots \leftarrow S_{k}\right),
$$

that is, $S_{j}^{\prime}=S_{j}$ for $j<t, S_{t}^{\prime}=R_{r+1}$ and $S_{j}^{\prime}=S_{j-1}$ for $t<j \leq k+1$. The arrow from $S_{t+1}^{\prime}=S_{t}$ to $S_{t}^{\prime}=R_{r+1}$ is $v_{t}$. The morphism (in Levtree) from $S_{*}^{\prime}$ to $R_{*}$ is defined in such a way that there is a commutative triangle

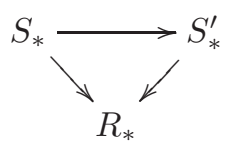

in Levtree, where the horizontal arrow (a morphism in $\operatorname{simp}(N F i n)$ ) is defined by the monotone injection $[k] \rightarrow[k+1]$ which omits $t$. Of course, $S_{t}^{\prime}$ should be mapped to $R_{r+1}=R_{u(t)}$ by the identity. This commutative triangle contributes to a sketchy description not only of $V$, but also of our preferred natural transformation from id to $V$. Now the functor $W$ is defined, on an object $S_{*} \rightarrow R_{*}$ of $\mathscr{U}$ as before, by starting from $V\left(S_{*} \rightarrow R_{*}\right)=\left(S_{*}^{\prime} \rightarrow R_{*}\right)$ as described and erasing from $S_{*}^{\prime}$ all the terms $S_{j+1}^{\prime}=S_{j}$ where $j \geq t$ and $v(j)=r+1$. Call the result $S_{*}^{\prime \prime} \rightarrow R_{*}$. Again there is a commutative triangle

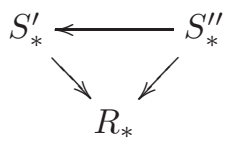

where the horizontal arrow (a morphism in $\operatorname{simp}(N F i n))$ is defined by a monotone injection $[k+1-a] \rightarrow[k+1]$ which omits an interval of the form $\{t+1, \ldots, t+a\}$. This contributes to a sketchy description not only of $W$, but also of our preferred natural transformation from $W$ to $V$. The morphism in $\mathscr{U}_{r}$ defined by this triangle is taken to a homotopy equivalence by the functor $\bar{Z}$. To illustrate that, here is a picture describing $S_{*}^{\prime}$ and $S_{*}^{\prime \prime}$ and the preferred morphism between them:
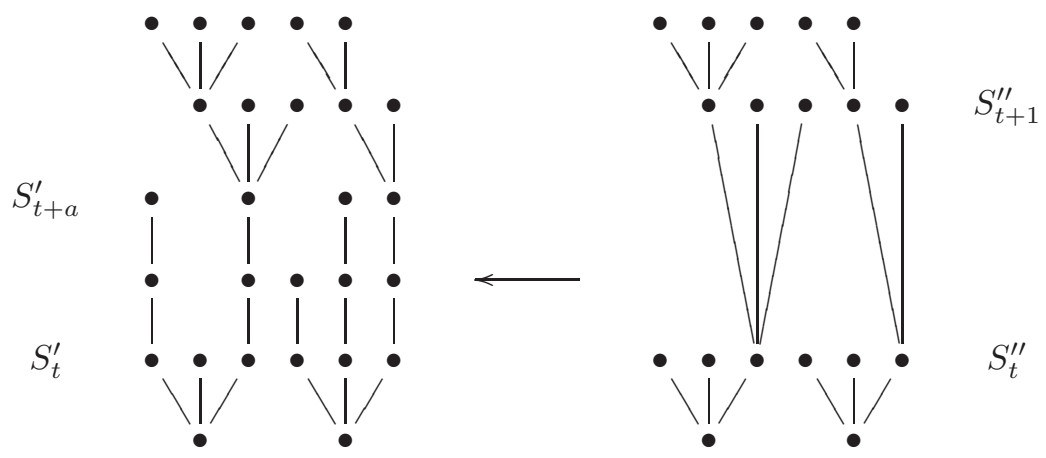

We are saying that the corresponding operator in $N_{d}^{r c} Q$ is a weak equivalence. This is based on the assumption that $Q(\underline{0})$ and $Q(\underline{1})$ are weakly contractible. 
Lemma 5.3. Let $Z=N_{d}^{r c} Q$. The homotopy unit $Z \rightarrow \mathbb{R} \xi_{*} \xi^{*} Z$ is a weak equivalence.

Proof. Let $T$ be an object of Tree ${ }^{\text {rc }}$. The standard formula for $\left(\mathbb{R} \xi_{*} \xi^{*} Z\right)(T)$ is

$$
\lim _{\xi\left(S_{*}\right) \rightarrow T} Z\left(\xi\left(S_{*}\right)\right) \text {. }
$$

Here the homotopy inverse limit is taken over a comma category $\mathscr{V}(T)$. An object of $\mathscr{V}(T)$ is an object $S_{*}$ of Levtree together with a morphism $f: \xi\left(S_{*}\right) \rightarrow T$ in Tree ${ }^{\text {rc }}$. A morphism in $\mathscr{V}(T)$ from $\left(S_{*}, f\right)$ to $\left(R_{*}, g\right)$ is a morphism $S_{*} \rightarrow R_{*}$ in Levtree which turns into a morphism over $T$ on applying $\xi$. Let $F_{T}$ from $\mathscr{V}(T)$ to spaces be the functor which takes $\left(S_{*}, f\right)$ to $Z\left(\xi\left(S_{*}\right)\right)$. Then we can write

$$
\left(\mathbb{R} \xi_{*} \xi^{*} Z\right)(T)=\text { holim } F_{T} .
$$

We proceed by induction on the number of nodes of $T$, where node means a vertex with more than one incoming edge. The induction beginning includes the case where $T$ has zero nodes. Then $T$ is linearly ordered. It is easy to see that $Z(T)$ is weakly contractible, since we are assuming weak contractibility of $Q(\underline{0})$ and $Q(\underline{1})$. Also, for each object $\left(S_{*}, f\right)$ in $\mathscr{V}(T)$, the space $Z\left(\xi\left(S_{*}\right)\right)=F_{T}\left(S_{*}, f\right)$ is weakly contractible since $\xi\left(S_{*}\right)$ is linearly ordered. Therefore, if $T$ has zero nodes, the unit map from $Z(T)$ to $\left(\mathbb{R} \xi_{*} \xi^{*} Z\right)(T)$ is a weak equivalence.

The induction beginning also includes the case where $T$ has exactly one node. This case is surprisingly hard. Let $\mathscr{V}_{0}(T)$ be the full subcategory of $\mathscr{V}(T)$ obtained by deleting all objects $\left(S_{*}, f\right)$ of $\mathscr{V}(T)$ where $\xi\left(S_{*}\right)$ has zero nodes, or equivalently, the sets $S_{i}$ all have cardinality $\leq 1$. It is easy to see that the restriction map

$$
\operatorname{holim} F_{T} \longrightarrow \operatorname{holim}\left(F_{T} \mid \mathscr{V}_{0}(T)\right)
$$

is a weak equivalence, since the value of $F_{T}$ on any object of $\mathscr{V}(T)$ not in $\mathscr{V}_{0}(T)$ is weakly contractible and since any morphism in $\mathscr{V}(T)$ with source in $\mathscr{V}_{0}(T)$ has target in $\mathscr{V}_{0}(T)$. Next, let $\mathscr{V}_{1}(T)$ be the full subcategory of $\mathscr{V}_{0}(T)$ consisting of those objects $\left(S_{*}, f\right)$ where the set $S_{0}$ has cardinality $>1$. (We write

$$
S_{*}=\left(S_{0} \leftarrow S_{1} \leftarrow \cdots \leftarrow S_{k-1} \leftarrow S_{k}\right)
$$

as usual.) The inclusion functor $\mathscr{V}_{1}(T) \rightarrow \mathscr{V}_{0}(T)$ has a right adjoint. (If $\left(S_{*}, f\right)$ in $\mathscr{V}_{0}(T)$ has $\left|S_{0}\right|=\left|S_{1}\right|=\cdots=\left|S_{j}\right|=1$ and $\left|S_{j+1}\right|>1$, then the value of that right adjoint on $\left(S_{*}, f\right)$ is obtained by deleting the terms $S_{0}, S_{1}, S_{2}, \ldots, S_{j}$.) Moreover the counit morphisms of the adjunction are taken to a weak equivalence by $F_{T}$. It follows that the restriction map

$$
\operatorname{holim}\left(F_{T} \mid \mathscr{V}_{0}(T)\right) \longrightarrow \operatorname{holim}\left(F_{T} \mid \mathscr{V}_{1}(T)\right)
$$

is a weak equivalence. Next, let $U \subset T$ be the set of incoming edges to the unique node of $T$. It has at least two elements by our assumption on $T$. Choose a total ordering on $U$. Let $\mathscr{V}_{2}(T) \subset \mathscr{V}_{1}(T)$ be the subcategory of $\mathscr{V}_{1}(T)$ defined as follows. An object $\left(S_{*}, f\right)$ of $\mathscr{V}_{1}(T)$ qualifies as an object of $\mathscr{V}_{2}(T)$ if $f$ takes the ordered set $S_{0}$ to $U$ by an order-preserving bijection. A morphism $\left(S_{*}, f\right) \rightarrow\left(R_{*}, g\right)$ in $\mathscr{V}_{1}(T)$ between such objects, given by a monotone map $u:[k] \rightarrow[\ell]$ and monotone injections $v_{j}: S_{j} \rightarrow R_{u(j)}$ for $j \in[k]$, qualifies as a morphism in $\mathscr{V}_{2}(T)$ precisely if $u(0)=0$. (In that case $v_{0}: S_{0} \rightarrow R_{0}$ must be an order preserving bijection, hence an identity map.) The inclusion of $\mathscr{V}_{2}(T)$ in $\mathscr{V}_{1}(T)$ has a left adjoint. Therefore the restriction map

$$
\operatorname{holim}\left(F_{T} \mid \mathscr{V}_{1}(T)\right) \longrightarrow \operatorname{holim}\left(F_{T} \mid \mathscr{V}_{2}(T)\right)
$$


is a weak equivalence. Now $F_{T}$ takes every morphism in $\mathscr{V}_{2}(T)$ to a weak equivalence of spaces. Moreover $\mathscr{V}_{2}(T)$ clearly has an initial object $\omega$. Together these properties imply that the projection from holim $\left(F_{T} \mid \mathscr{V}_{2}(T)\right)$ to $F_{T}(\omega)$ is a weak equivalence. Putting all that together, it follows that the projection from holim $F_{T}$ itself to $F_{T}(\omega)$ is a weak equivalence. Then it follows easily by inspection that the unit map $Z(T) \rightarrow\left(\mathbb{R} \xi_{*} \xi^{*} Z\right)(T)=$ holim $F_{T} \simeq F_{T}(\omega)$ is a weak equivalence. This completes our discussion of the case where $T$ has exactly one node.

We come to the induction step, which is rather formal and uses a mildly sheaf theoretic argument. Suppose that $T$ is an object of Tree ${ }^{\text {rc }}$ such that $T=T_{0} \cup T_{1}$ where $T_{0}$ and $T_{1}$ are subtrees (by which, at this point, we simply mean sub-posets) of $T$. More precisely we require that if $e \in T_{0}$ and $e^{\prime} \in T$ with $e^{\prime} \leq e$, we have $e^{\prime} \in T_{0}$, and similarly for $T_{1}$. We also require that if $e, e^{\prime} \in T$ and both are incoming edges for the same vertex, then either $e, e^{\prime}$ are both in $T_{0}$ or both not in $T_{0}$; and similarly for $T_{1}$. The following picture is an example.

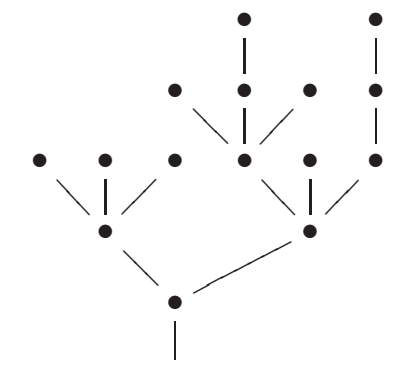

$T$

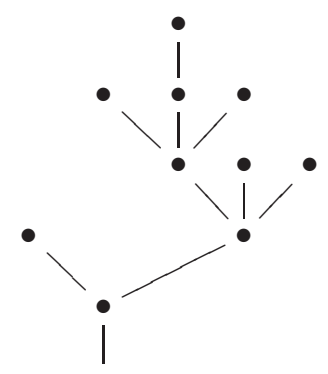

$T_{0}$

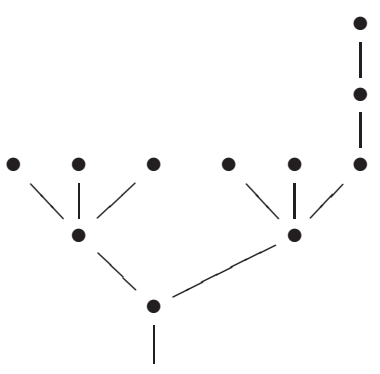

$T_{1}$

Then it is easy to see that the square of inclusion-induced maps

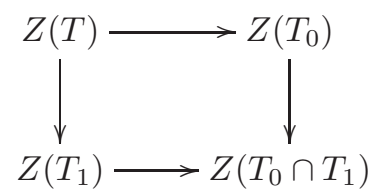

is a homotopy pullback square. If we can show that the square of inclusion-induced maps

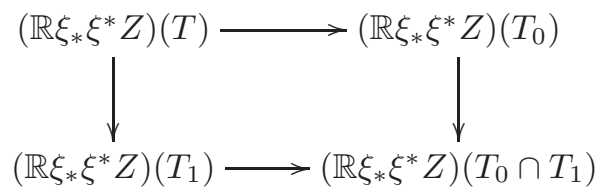

is also a homotopy pullback square, then that can pass for the induction step. With the abbreviations or translations above, we have $\left(\mathbb{R} \xi_{*} \xi^{*} Z\right)(T)=$ holim $F_{T}$ where $F_{T}$ is defined on $\mathscr{V}_{T}$. Let $\mathscr{V}_{T}^{\prime}$ be the full subcategory of $\mathscr{V}_{T}$ consisting of all objects

$$
\left(S_{*}, f: \xi\left(S_{*}\right) \rightarrow T\right)
$$


such that $\xi\left(S_{*}\right)$ lands in $T_{0}$ or in $T_{1}$ or even in $T_{0} \cap T_{1}$. By inspection, the square of restriction maps

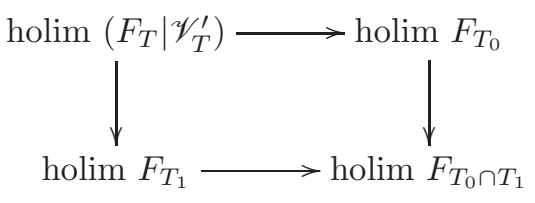

is a homotopy pullback square. Therefore it is enough to show that the restriction map from holim $F_{T}$ to holim $\left(F_{T} \mid \mathscr{V}_{T}^{\prime}\right)$ is a weak equivalence. A formula of DwyerKan [4, 9.7] or Bousfield-Kan allows us to identify holim $\left(F_{T} \mid \mathscr{V}_{T}^{\prime}\right)$ with the homotopy inverse limit of $\mathbb{R} \eta_{*}\left(F_{T} \mid \mathscr{V}_{T}^{\prime}\right)$, where $\eta: \mathscr{V}_{T}^{\prime} \rightarrow \mathscr{V}_{T}$ is the inclusion and $\mathbb{R} \eta_{*}$ denotes the homotopy right Kan extension along $\eta$. In the definition of $\mathbb{R} \eta_{*}$ we use, for each object $\left(S_{*}, f\right)$ in $\mathscr{V}_{T}$, the comma category $\eta /\left(S_{*}, f\right)$. In that comma category there is a diagram (of three objects)

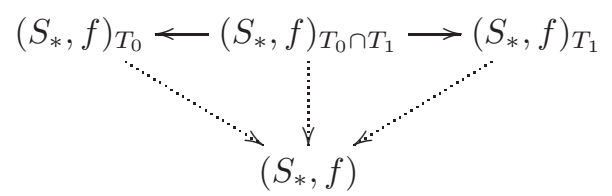

where the subscripts indicate evident pullback operations; for example $\left(S_{*}, f\right)_{T_{0}}$ is made from the portion of $S_{*}$ taken to $T_{0}$ by $f$. That diagram can be viewed as a subcategory of $\eta /\left(S_{*}, f\right)$. It is a terminal subcategory in the sense that the inclusion functor has a left adjoint. Hence the value of $\mathbb{R} \eta_{*}\left(F_{T} \mid \mathscr{V}_{T}^{\prime}\right)$ at the object $\left(S_{*}, f\right)$ of $\mathscr{V}_{T}$ can be identified with the homotopy pullback of

$$
F_{T}\left(\left(S_{*}, f\right)_{T_{0}}\right) \rightarrow F_{T}\left(\left(S_{*}, f\right)_{T_{0} \cap T}\right) \leftarrow F_{T}\left(\left(S_{*}, f\right)_{T_{1}}\right)
$$

which in turn can be identified with $F_{T}\left(S_{*}, f\right)=Z\left(\xi\left(S_{*}\right)\right)$ by the sheaf property of $Z$. This completes the proof.

It is very easy to show (again) that the map (4.1) is a weak equivalence using lemmas 5.2 and 5.3. Indeed, lemma 5.2 implies that the standard map

$$
\mathbb{R m a p}\left(\xi^{*} N_{d}^{r c} P, \xi^{*} N_{d}^{r c} Q\right) \longrightarrow \mathbb{R} \operatorname{map}\left(\alpha^{*} \xi^{*} N_{d}^{r c} P, \alpha^{*} \xi^{*} N_{d}^{r c} Q\right)
$$

is a weak equivalence and lemma 5.3 implies that the standard map

$$
\mathbb{R} \operatorname{map}\left(N_{d}^{r c} P, N_{d}^{r c} Q\right) \longrightarrow \mathbb{R} \operatorname{map}\left(\xi^{*} N_{d}^{r c} P, \xi^{*} N_{d}^{r c} Q\right)
$$

is a weak equivalence. See [1, lem.A.1] and remember diagram (5.2). This new proof carries over with a little mutatis mutandis to show that the map (4.2) is also weak equivalence. In this way we have completed the proof of theorem 3.1] since that had already been reduced to showing that (4.2) is a weak equivalence.

\section{REFERENCES}

[1] P. Boavida de Brito and M.S. Weiss, Spaces of smooth embeddings and configuration categories, arXiv:1502.01640 (to appear in J. Topol.)

[2] D.-C. Cisinski and I.Moerdijk, Dendroidal sets as models for homotopy operads, J. Topol. 4 (2011), 257-299.

[3] D.-C. Cisinski and I.Moerdijk, Dendroidal Segal spaces and $\infty$-operads, J. Topol. 6 (2013), 675-704.

[4] W. G. Dwyer and D. Kan, A classification theorem for diagrams of simplicial sets, Topology 23 (1984), 139-155. 
[5] W. G. Dwyer and D. Kan, Function complexes in homotopical algebra, Topology 19 (1980), 427-440.

[6] P. Goerss and K. Schemmerhorn, Model categories and simplicial methods, Interactions between homotopy theory and algebra, 3-49, Contemp. Math. vol. 436, Amer.Math.Soc., Providence, RI, 2007.

[7] I. Moerdijk and I. Weiss, Dendroidal sets, Algebr. Geom. Topol. 7 (2007), 1441-1470.

[8] C. Rezk, A model for the homotopy theory of homotopy theory, Trans.Amer.Math.Soc. 353 (2001), 973-1007.

[9] A. Voronov, Notes on universal algebra, Graphs and patterns in mathematics and theoretical physics, 81-103, Proc.Sympos.Pure Math. vol.73, Amer.Math.Soc., Providence, RI, 2005.

Math.Institut, WWU Münster, 48149 Münster, Einsteinstrasse 62, Germany

E-mail address: m.weiss@uni-muenster.de 